\title{
QUEEN'S
UNIVERSITY
BELFAST
}

\section{A Low Radar Cross Section Dipole Antenna Simulation}

Machado, G. G., Cahill, R., \& de Melo, M. T. (2015). A Low Radar Cross Section Dipole Antenna Simulation. In Proceedings of the International Microwave and Optoelectronics Conference (IMOC 2015) (pp. 1-5). IEEE MTT S. https://doi.org/10.1109/IMOC.2015.7369189

\section{Published in:}

Proceedings of the International Microwave and Optoelectronics Conference (IMOC 2015)

\section{Document Version:}

Peer reviewed version

\section{Queen's University Belfast - Research Portal:}

Link to publication record in Queen's University Belfast Research Portal

\section{Publisher rights}

( 2015 IEEE. Personal use of this material is permitted. Permission from IEEE must be obtained for all other uses, in any current or future media, including reprinting/republishing this material for advertising or promotional purposes, creating new collective works, for resale or redistribution to servers or lists, or reuse of any copyrighted component of this work in other works.

\section{General rights}

Copyright for the publications made accessible via the Queen's University Belfast Research Portal is retained by the author(s) and / or other copyright owners and it is a condition of accessing these publications that users recognise and abide by the legal requirements associated with these rights.

\section{Take down policy}

The Research Portal is Queen's institutional repository that provides access to Queen's research output. Every effort has been made to ensure that content in the Research Portal does not infringe any person's rights, or applicable UK laws. If you discover content in the Research Portal that you believe breaches copyright or violates any law, please contact openaccess@qub.ac.uk. 


\title{
A Low Radar Cross Section Dipole Antenna Array Simulation
}

\author{
G.G. Machado \\ School of Electronics, Electrical Engineering and Computer Science \\ Queen's University Belfast \\ Federal University of Pernambuco \\ email: gabriel.gm91@gmail.com \\ R. Cahill \\ The Institute of Electronics, Communications and Information Technology (ECIT) \\ Queen's University Belfast \\ University Road, Belfast BT9 1NN, Northern Ireland \\ M.T. de Melo \\ Federal University of Pernambuco \\ Av. Academico Helio Ramos, S/N, 50.740-530, Brazil
}

\begin{abstract}
This paper reports a new method for reducing the Radar Cross-Section (RCS) of a metal backed dipole antenna. Numerical simulations are used to show that when the Perfect Electrical Conductor (PEC) is replaced by a carefully designed Frequency Selective Surface (FSS), the electromagnetic performance of the antenna is similar in band, but the RCS of the structure is significantly lower out of band. The design of the FSS and the return loss, radiation patterns and RCS are presented for an antenna which operates at a center frequency of $4 \mathrm{GHz}$ and the results are compared with a conventional metal backed arrangement.
\end{abstract}

Keywords-Low RCS; Radar Cross-section; Dipole Antenna; Frequency Selective Surface; FSS; Jerusalem Cross

\section{INTRODUCTION}

As demands for the stealth budget in warfare increases, significant investment is being made to develop techniques capable of providing electromagnetic cloaking of vehicles and equipment used in defence systems. Significant research has been published in the open literature which shows that radar backscatter suppression can be achieved using thin carbon based microwave absorbers, resistive metamaterials, and advanced surface treatments such as nano composite paint that is used for the F-117 Nighthawk. However some applications require structures that exhibit a strong frequency selective RCS behavior so that low visibility to radar is obtained only in one or two defined bands, but not at all frequencies. For example antennas based on dipole array configurations are often used for communication systems, and for these structures a $3 \mathrm{~dB}$ increase in gain is often desirable and achieved simply by placing the radiating elements $\approx \lambda / 4$ above a metal ground plane. At normal incidence the RCS is very large and proportional to the dimensions of the reflector; but for this application the backing structure must strongly reflect signals from the dipoles over the operating frequency range of the antenna. However at frequencies, above and below the operating band, the electromagnetic behavior of the ground plane can be modified to provide a low radar signature. In this paper we show that by replacing the metal backing structure with a carefully designed FSS, a significant reduction in the out of band RCS is achieved [1], [2], but in band the resonant behavior of the periodic array yields similar return loss results and radiation patterns for the dipole antenna.

\section{Methodology}

For this feasibility study a half wavelength linear dipole antenna was chosen because of its simplicity and well known radiation patterns [3]. By defining the operating bandwidth using the metric VSWR 2, the frequency range between $3.42 \mathrm{GHz}$ and $4.86 \mathrm{GHz}(35 \%)$ was obtained from the CST Microwave Studio EM simulator [4] by varying the width and gap between the dipole arms.

Subsequently simulations for the dipole above infinite and different size metal plates was performed to find the optimum separation in terms of the impedance match and shape of the bi-directional radiation patterns at $4 \mathrm{GHz}$, the center operating frequency of the antenna.

The FSS design was based on Jerusalem Cross elements which exhibit identical spectral responses for vertical and horizontal polarized waves at normal incidence [5], [6]. The reflection band of the FSS was centered at $4 \mathrm{GHz}$, and simulations were made using the physical and electrical characteristics of a commercially available low loss microwave substrate on which the elements are printed. For each case the dimensions of the unit cells were adjusted to obtain a $90 \%$ reflectivity bandwidth of $35 \%$.

In the EM simulator the metal plate was replaced by a finite size FSS $(210 \mathrm{~mm} \times 210 \mathrm{~mm})$ with the same dimensions, which reflects $>90 \%$ of the energy radiated by the dipole in the lower hemisphere. For this arrangement the return loss, gain and radiation pattern performance are compared at the center and the upper and lower band edges. 
Finally RCS simulations were performed for both antenna configurations from $0 \mathrm{GHz}$ to $7 \mathrm{GHz}$ and by comparing the results we demonstrate the increase in functionality that is obtained by deploying the FSS. Fig. (1) depicts a flow chart which summarizes the methodology that was used in this feasibility study

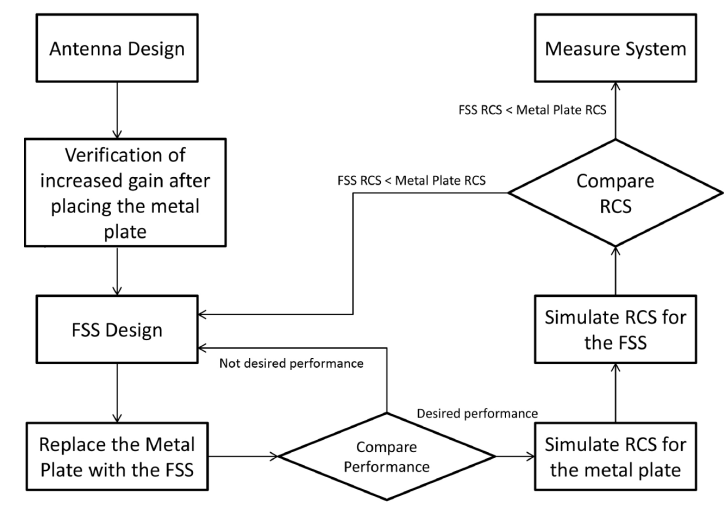

Fig. 1: Flow chart illustrating design methodology

\section{Simulation Preparation}

\section{A. Antenna Design}

The antenna design required a parametric study to achieve the desired bandwidth which is defined by the frequency range over which the VSWR 2. The key geometric parameters are the length of the dipole which is employed to adjust the frequency of operation, and the diameter of the arms which is varied to obtain the bandwidth [7]. Fine tuning of the resonant frequency is obtained by choosing the dimensions of the physical gap between the elements. The length and width of the optimised dipole design are $37.47 \mathrm{~mm} \mathrm{~mm}$ and $4 \mathrm{~mm}$ respectively.

Bidirectional radiation is obtained when the dipole is placed $\lambda / 4$ above an infinite size metal plate. Since at this distance the short circuit exhibited by the backing structure is transformed to an open circuit at the input port to the dipole, the impact on the input impedance is negligible. Fig. (2) shows the simplified case for a horizontally oriented dipole placed above an infinite metal sheet. The analysis is based on image theory.

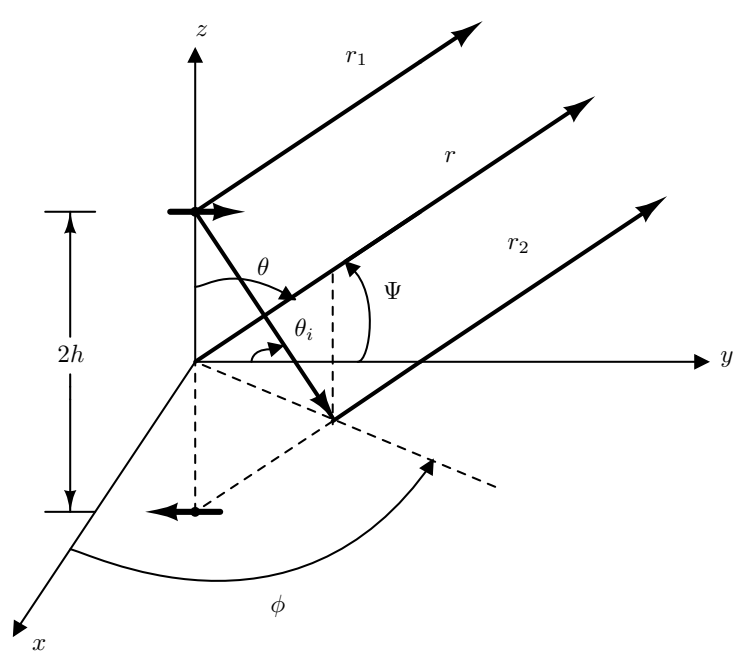

Fig. 2: Horizontal Dipole (represented by the arrow) above a distance $h$ from the ground plane

However, as the CST simulator has the ability to analyze a non-ideal environment, which is the case for finite size ground planes, stray capacitances and edge scattering can be accounted for in the numerical simulations. Thus, a parametric study was performed to find the optimum separation between the dipole and the reflector. The dimensions of the metal plate area $(210 \mathrm{~mm} \times 210 \mathrm{~mm})$ were obtained by determining the smallest area possible which would produce a maximum gain reduction smaller than $0.2 \mathrm{dBi}$, when compared to an infinite ground plane.

\section{B. FSS Design}

The main purpose of using a FSS to replace the metal reflector is the ability to tailor the spectral response so that at resonance and at all frequencies within the $35 \%$ bandwidth obtained for the dipole antenna, the electromagnetic behavior exhibited by the periodic array is similar to a metal plate. But at other frequencies the magnitude of the current which flows in the Jerusalem cross elements is very low so the FSS appears to be invisible to incident microwave signals and therefore presents a low RCS out of band. For an ideal FSS design, visibility to radar is therefore attributed to scattering from the FSS in band and the dipole elements out of band. For our case the structure can be engineered to present a low RCS at all frequencies outside the $35 \%$ operating band of the dipoles. A preliminary estimate of the physical dimensions of the unit cell geometry can be obtained from (1) [8], where $\lambda_{r}$ is the wavelength at resonance $(4 \mathrm{GHz})$. The other geometrical parameters are illustrated in Fig. (3).

$$
\lambda_{r}=2 \sqrt{d p \ln \left(\frac{2 p}{\pi w}\right) \ln \left(\frac{2 p}{\pi g}\right)}
$$




\section{Simulation Results and Discussion}

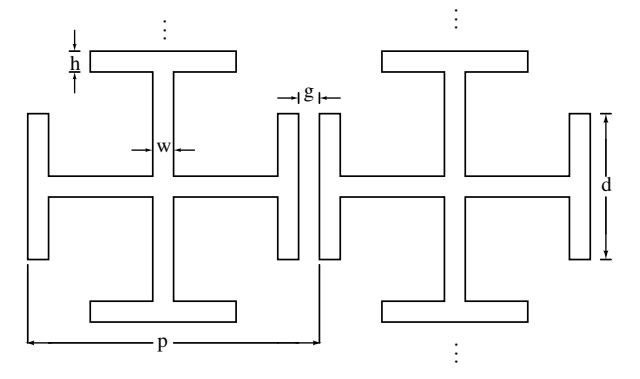

Fig. 3: Structure and parameters for the designed Jerusalem Cross, where $p=10.5 \mathrm{~mm}, d=6 \mathrm{~mm}, w=h=0.1 \mathrm{~mm}$, $g=90 \mu m, t=18 \mu m$.

The computed spectral response of a single layer FSS printed on the $18 \mu \mathrm{m}$ thick microwave substrate shows that the $-0.5 \mathrm{~dB}$ reflectivity bandwidth is very much narrower than the $35 \%$ target value centered $4 \mathrm{GHz}$, and therefore to achieve the same bandwidth as the dipole antenna a second identical doubly periodic FSS layer was employed in the ground plane design [5]. In this way the structure shown in Fig. (4), permits the phase response of the individual arrays to be controlled so as to generate a wider reflection band and a fast transmission roll off below and above resonance which is desirable in order to achieve a low RCS outside the $3.42 \mathrm{GHz}$ and $4.86 \mathrm{GHz}$ frequency range. For this study the separation between two FSS was selected to be multiples of $\lambda / 4$ spacing.

The FSS was printed on a Rogers RT5880 substrate with $\epsilon_{r}=2.2, \tan (\delta)=0.0009$ and a thickness of $0.254 \mathrm{~mm}$.

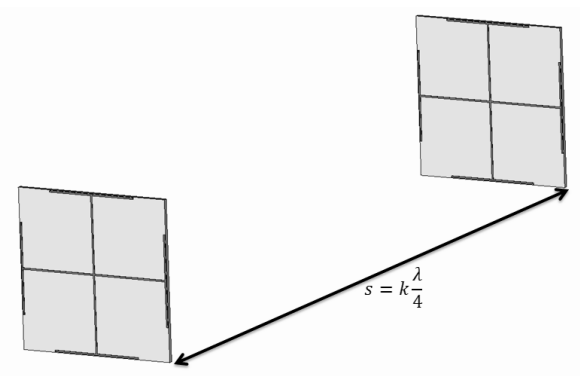

Fig. 4: Single cell representation for a double layer FSS on Microwave Studio

\section{RCS Simulation}

RCS simulations were made between $0 \mathrm{GHz}$ and $7 \mathrm{GHz}$ to study the radar backscatter from the metal plate and the FSS backed antenna arrangements. For both cases the plate size was $210 \mathrm{~mm}$ X $210 \mathrm{~mm}$. The results obtained from the two simulations were compared to confirm that the FSS exhibits a lower RCS then the metal plate out of band. Only normal incident waves were considered in the CST macro for monostatic RCS calculations.

\section{A. Antenna and Metal Plate Configuration}

The predicted return loss results for the antenna and metal plate configuration are depicted in Fig. (5), for 3 different plate sizes.

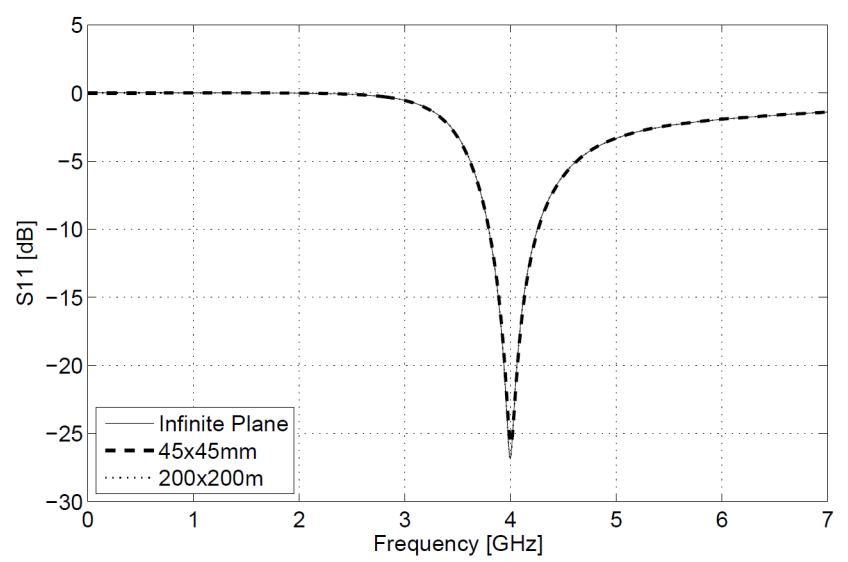

Fig. 5: Simulated return loss of antenna above metal ground plane

The computed peak gain for the single dipole antenna placed above an infinite metal ground plane is $7.4 \mathrm{dBi}$ at $4 \mathrm{GHz}$ whereas a gain of $7.2 \mathrm{dBi}$ is predicted for a reflector size of $210 \times 210 \mathrm{~mm}$. The E plane radiation pattern shown in Fig. (6) is largely bidirectional with a front to back ratio of about $20 \mathrm{~dB}$ due to the fact that the metal plate has a finite size.

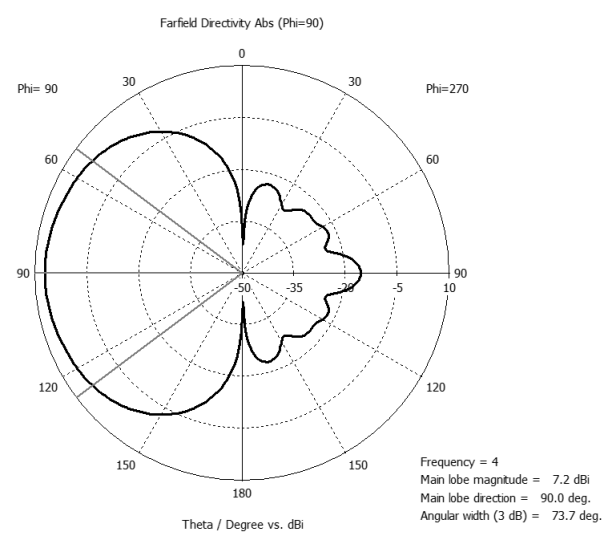

Fig. 6: Simulated radiation pattern of antenna above a $210 \mathrm{~mm}$ $\mathrm{X} 210 \mathrm{~mm}$ metal ground plane at $4 G H z$.

\section{B. Antenna and FSS configuration}

Because a single layer FSS design does not satisfy the bandwidth criteria, a parametric study was conducted for a two layer FSS with dimensions given in Fig. (3), for different distances between the two periodic layers. The separation distance was based on multiples of the desired center rejection wavelength, as shown in Fig. (4). The results are shown in Fig. (7). 


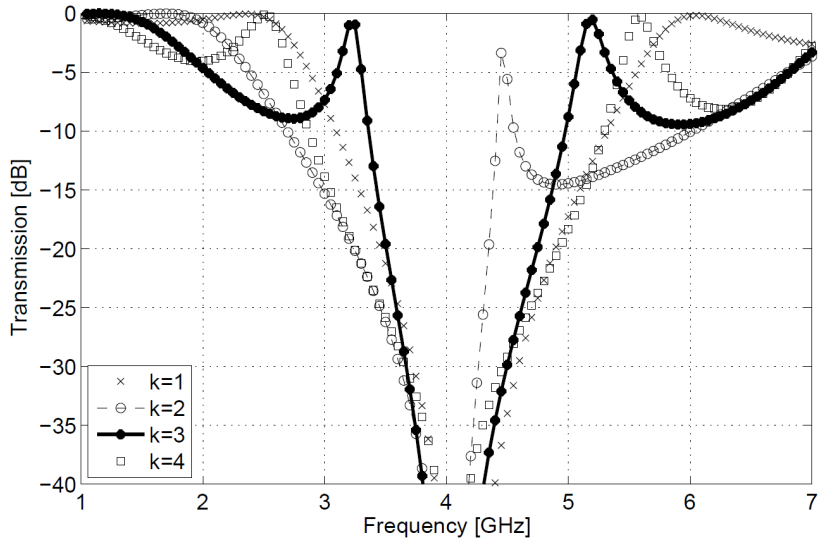

Fig. 7: Simulated spectral response of a two layer FSS transmission for four different separation distances

For a separation distance of $3 / 4 \lambda$, the $-10 \mathrm{~dB}$ transmission response matches exactly the antenna bandwidth, but as shown in Fig. (7) the predicted transmission at $3 \mathrm{GHz}$ and $6 \mathrm{GHz}$ is close to $-10 \mathrm{~dB}$, therefore the RCS reduction would be small given that $90 \%$ of the incident power is reflected from the FSS at these frequencies. However this arrangement is preferable to the other three options studied, since these provide a reflection bandwidth that is either too small or larger than desired. The representation of the system is shown in Fig. (8). The simulated radiation patterns at the center frequency and band edges for the metal and FSS backed dipoles are shown in the Figs (911).

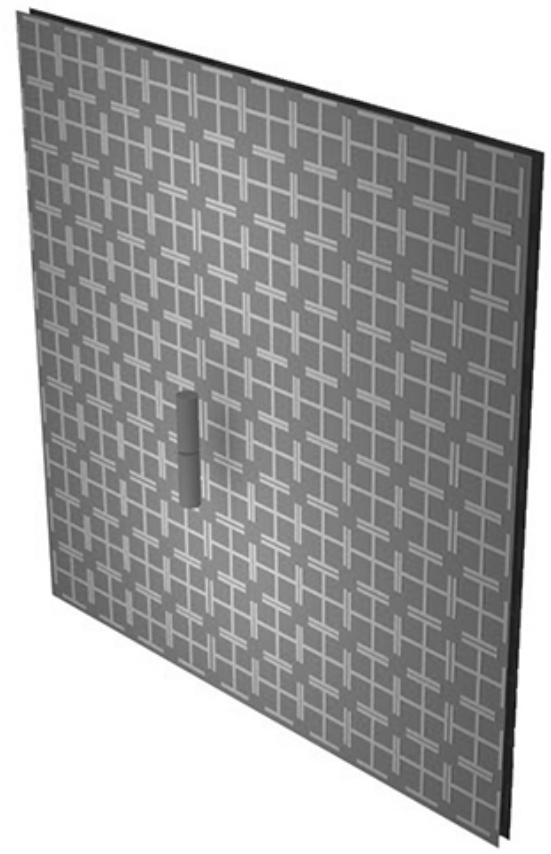

Fig. 8: Dipole antenna and the two layers FSS representation.

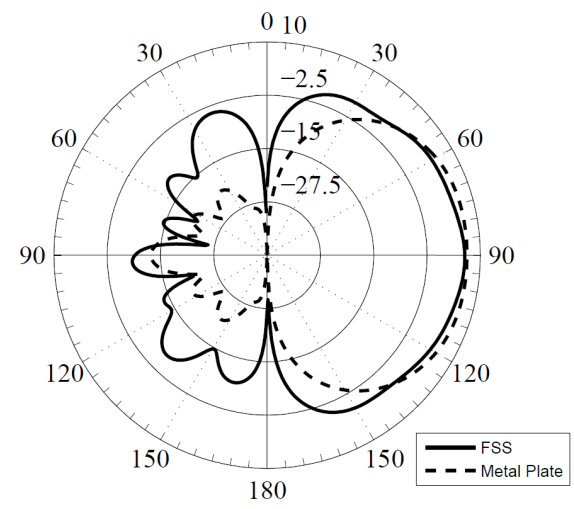

Fig. 9: Simulated E plane radiation pattern for the metal and FSS backs dipole at $3.5 \mathrm{GHz}$

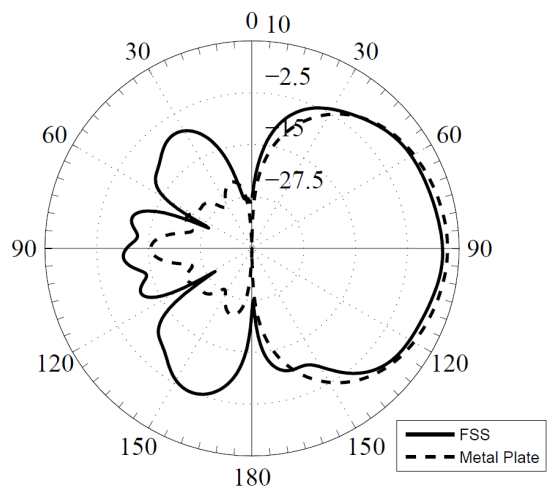

Fig. 10: Simulated E plane radiation pattern for the metal and FSS backs dipole at $4.0 \mathrm{GHz}$

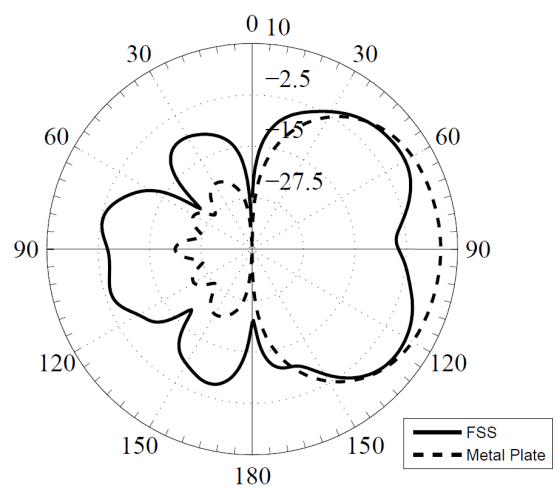

Fig. 11: Simulated E plane radiation pattern for the metal and FSS backs dipole at $4.85 \mathrm{GHz}$

Excellent agreement is shown at 3.5 and $4.0 \mathrm{GHz}$ thus demonstrating that the electromagnetic behavior of the FSS is similar to a perfect electric conductor at these frequencies. At $4.85 \mathrm{GHz}$ an approximated $5 \mathrm{~dB}$ null is observed at boresight which is attributed to destructive interference between the 
energy in the upper hemisphere and signals reflected from the FSS. As a general observation it is observed that for all three cases the energy radiated in the backlobes is higher when the antenna is backed by a FSS, however the reduction in the peak gain is less than $0.2 \mathrm{~dB}$ at $4 \mathrm{GHz}$.

\section{RCS Results}

As previously stated, the main objective of the study was to design an antenna reflector which simultaneously exhibits a peak antenna gain similar to a metal plate, and a low radar cross section at higher and lower frequencies.

CST was employed to simulate the RCS of a $210 \times 210$ $\mathrm{mm}$ metal plate and the results were verified by comparing these with (2), which is based on classical theory [9]

$$
\sigma=\frac{4 \pi A^{2}}{\lambda^{2}}
$$

In the above equation, $\mathrm{A}$ is the area of the plate, $A$ is the wavelength of the waves, and $\sigma$ is the RCS. Theoretical results derived from the equation above and the simulation results are plotted and compared in Fig. (12).

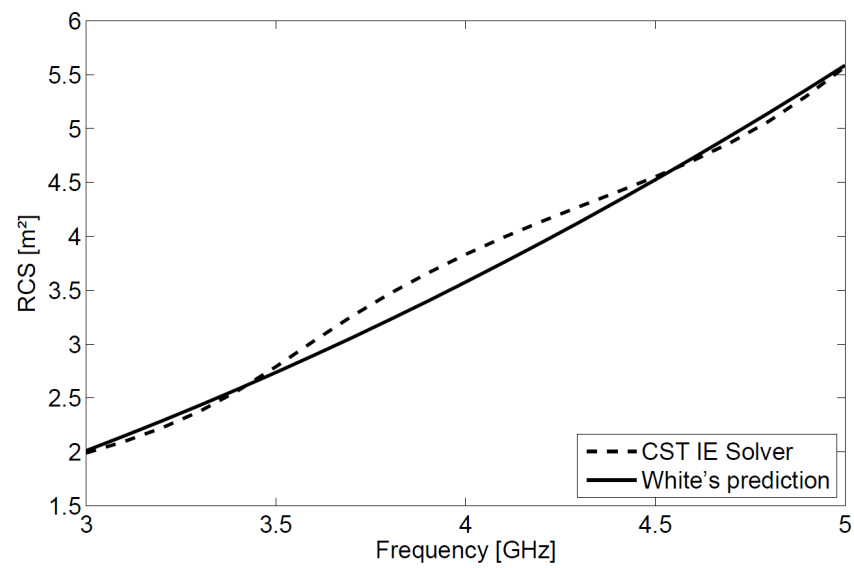

Fig. 12: Simulated RCS of a 210 × $210 \mathrm{~mm}$ metal plate obtained from CST and eqn (2)

Close correlation between the two plots is shown thereby confirming the accuracy of CST for solving this type of problem. The EM solver was then employed to compare the RCS of the metal plate and a $210 \times 210 \mathrm{~mm}$ two layer FSS. As shown in Fig. (13), the FSS exhibits a significantly lower RCS than the metal plate particularly above the upper edge of the operating band of the dipole antenna.

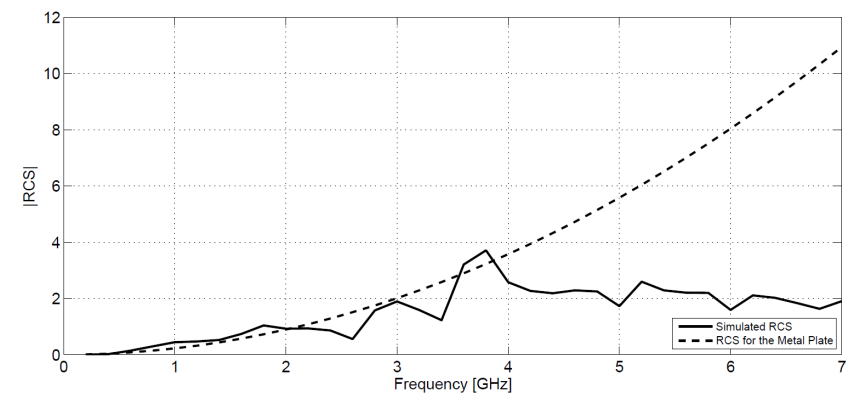

Fig. 13: Comparison between the FSS and Metal Plate RCS prediction

\section{CONClusion}

The design of a dipole antenna and FSS were performed and the results integrated to study how the entire system behaves in terms of radiation pattern performance and radar cross section. It was shown that the RCS for this configuration is lower than a conventional arrangement with the antenna placed above a metal ground plane, whereas the in band gain is very similar. Therefore this design approach opens up many new possibilities for controlling the visibility to radar of flat plate antennas.

\section{REFERENCES}

[1] N. Misran, R. Cahill, and V. Fusco, "Rcs reduction technique for reflectarray antennas," Electronics Letters, vol. 39, no. 23, pp. 1630-2-, Nov 2003.

[2] "Use of frequency selective surfaces for the reduction of radar cross ssection of antennas and scattering objects," http://utopia.duth.gr/ iaitidis/ESA\%20conference\%202010/Papers/ session\%207/FCXNL-10C09-1988164-1-1988164Monorchio.pdf, accessed: 09-12-2014.

[3] C. A. Balanis, Antenna Theory: Analysis and Design. Wiley, 2012.

[4] “Cst - computer simulation technology," https://www.cst.com, accessed: January 2013.

[5] R. Dickie, R. Cahill, V. Fusco, H. Gamble, and N. Mitchell, "Thz frequency selective surface filters for earth observation remote sensing instruments," Terahertz Science and Technology, IEEE Transactions on, vol. 1, no. 2, pp. 450-461, Nov 2011.

[6] B. Munk, Frequency Selective Surfaces: Theory and Design. Wiley, 2005. [Online]. Available: http://books.google.co.uk/books? id=9pNMhRQrpScC

[7] R. Johnson and H. Jasik, Antenna Engineering Handbook, 3rd ed. McGraw-Hill Professional,, 1992.

[8] I. Anderson, "On the theory of self-resonant grids," Bell System Technical Journal, The, vol. 54, no. 10, pp. 1725-1731, Dec 1975.

[9] M. O. White, "Radar cross-section: measurement, prediction and control," Electronics Communication Engineering Journal, vol. 10, no. 4, pp. 169-180, Aug 1998. 